\title{
Research Article \\ Effect of Various Ring Yarns on Fabric Comfort
}

\author{
Mukesh Kumar Singh and Akansha Nigam \\ Uttar Pradesh Textile Technology Institute, Souterganj, Kanpur 208001, India \\ Correspondence should be addressed to Mukesh Kumar Singh; mukesh70ster@gmail.com
}

Received 5 September 2012; Revised 8 July 2013; Accepted 29 July 2013

Academic Editor: Paul C. Xirouchakis

Copyright (C) 2013 M. K. Singh and A. Nigam. This is an open access article distributed under the Creative Commons Attribution License, which permits unrestricted use, distribution, and reproduction in any medium, provided the original work is properly cited.

Comfort performance of woven structures made of various types of ring spun yarns like carded, combed, and compact spun yarns has been reported in the present study. Carded, combed, and compact spun yarns are entirely different in structure in terms of fibre migration inside the yarn body, level of free space inside the yarn, number of hairs, and length of hairs on yarn surfaces. In this study, $197 \mathrm{dtex}$ and $144 \mathrm{dtex}(30 \mathrm{~s} \mathrm{Ne}$ and $40 \mathrm{~s} \mathrm{Ne}$ ) ring spun combed yarns are used as a warp. The same cotton mixing was used to manufacture $30 \mathrm{~s} \mathrm{Ne}$ and $40 \mathrm{~s} \mathrm{Ne}$ carded, combed, and compact yarns. Both $30 \mathrm{~s}$ and $40 \mathrm{~s}$ Ne linear density yarns were prepared by all three carded, combed, and compact yarn manufacturing routes. The structure of fibre strand in filling yarn has a great impact on comfort related properties, that is, thermal conductivity, $q_{\max }$, air permeability, wicking, and moisture vapour permeability.

\section{Introduction}

Can yarn engineering influence the comfort management of woven fabric? Yes, because yarn engineering changes the configuration of constituent fibres in yarn body. Comfort is qualitative attribute, and it is one of the most demanding aspects from clothing customers of present era. Comfort is a collective feeling generated by summation of impulses sent to the nerves from different receptors in the human brain [1]. Psychological, tactile, and thermal comforts are three classes of comfort. Transmission ability of fabric is related to the thermal comfort because it maintains the temperature and moisture level at skin through transmission of heat and sweat generated by human body. Majority of scientists agreed on the point that transmission of air, heat, and water is probably the most important factors in clothing comfort $[2,3]$.

The comfort performance of clothing depends on the structure and properties of fibre and yarn used. Alteration in yarn structure is able to make some revolutionary changes in fabric quality from comfort point of view. More than 70 percent of yarns are still ring spun yarns used for clothing purposes. Carded, combed, and compact are three different routes to manufacture ring spun yarns. As the yarn structure changes in carded, combed, and compact spun yarns, various types of external and internal changes take place in resultant yarns. Fibre configurations remain different in carded, combed, and compact yarns. Hence, a systematic research is required in this field to understand the role of ring yarn structure on fabric comfort. In order to investigate the effect of ring yarn type on fabric comfort, a focused research is required.

The sole object of this study is to find out the comfort performance of woven fabric samples made out of carded, combed, and compact spun yarns.

\section{Purpose}

Most of the shirting fabrics are made of spun yarns which is the most comfort demanding segment of textiles clothing. By opting systematic changes in yarn structure by engineering the yarn, comfort aspect can be managed up to some extent. This concept is the major source of inspiration in this study.

\section{Material and Methods}

Twelve $100 \%$ cotton plain woven fabrics were manufactured on airjet loom with pick density 88 and 108 for all three carded, combed, and compact spun ring yarns and two different weft counts 30 s and 40 s Ne using 40 s Ne combed warp thread. The warp density in weavers beam 110 per inch was kept for all twelve samples. The nomenclature and details of fabric set are given in Table 1. 
TABLE 1: Cotton fabric sample details.

\begin{tabular}{|c|c|c|c|c|}
\hline S. no. & Sample Code & Sample detail & Thickness (mm) & Fabric GSM \\
\hline 1 & $30 \mathrm{CaF} 88$ & Compact warp count 40 s Ne, Ne, EPI 120, carded weft count 30 s Ne, PPI 88 & 0.348 & 115 \\
\hline 2 & 30CaF108 & Compact warp count 40s Ne, Ne, EPI 120, carded weft count 30s Ne, PPI 108 & 0.357 & 122 \\
\hline 3 & $40 \mathrm{CaF} 88$ & Compact warp count 40 s Ne, Ne, EPI 120, carded weft count 40s Ne, PPI 88 & 0.341 & 110 \\
\hline 4 & 40CaF108 & Compact warp count 40 s Ne, Ne, EPI 120, carded weft count 40s Ne, PPI 108 & 0.346 & 115 \\
\hline 5 & $30 \mathrm{CoF} 88$ & Compact warp count 40 s Ne, Ne, EPI 120, combed weft count 30 s Ne, PPI 88 & 0.334 & 117 \\
\hline 6 & 30CoF108 & Compact warp count 40s Ne, Ne, EPI 120, combed weft count 30s Ne, PPI 108 & 0.339 & 123 \\
\hline 7 & $40 \mathrm{CoF} 88$ & Compact warp count 40 s Ne, Ne, EPI 120, combed weft count 40 s Ne, PPI 88 & 0.329 & 115 \\
\hline 8 & 40CoF108 & Compact warp count 40s Ne, Ne, EPI 120, combed weft count 40s Ne, PPI 108 & 0.336 & 120 \\
\hline 9 & $30 \mathrm{CtF} 88$ & Compact warp count 40 s Ne, Ne, EPI 120, compact weft count 30s Ne, PPI 88 & 0.328 & 119 \\
\hline 10 & 30CtF108 & Compact warp count 40s Ne, Ne, EPI 120, compact weft count 30s Ne, PPI 108 & 0.331 & 123 \\
\hline 11 & $40 \mathrm{CtF} 88$ & Compact warp count 40 s Ne, Ne, EPI 120, compact weft count 40 s Ne, PPI 88 & 0.326 & 115 \\
\hline 12 & $40 \mathrm{CtF} 108$ & Compact warp count 40s Ne, Ne, EPI 120, compact weft count 40s Ne, PPI 108 & 0.329 & 124 \\
\hline
\end{tabular}

3.1. Fabric Characteristics. Fabric set of fabric samples was measured with the help of counting glass according to ASTM D3775-03 standard. Fabric thickness was calculated as per the ASTM D1777-96 standard using Paramount thickness tester at a pressure of $20 \mathrm{gf} / \mathrm{cm}^{2}$ with an accuracy of $0.01 \mathrm{~mm}$. Fabric areal density and yarn linear density were determined according to ASTM D1059 standard. An average of 20 observations is reported here. Fabric and yarn pictures are captured by Kyowa ME-POL2 microscope at magnification of 40.

3.2. Thermal Behaviour. The measurements of thermal behaviour of finished fabrics made of different types of filling yarns with various linear densities were performed with the help of ALAMBETA instrument constructed in Czech Republic. Various researchers have accepted the importance of thermal contact, in order to ensure thermal resistance of a fabric and found Alambeta a suitable instrument. Ten measurements were made on both face and back sides of the fabric in four-layer form. The arithmetic mean of twenty observations is reported here for each sample.

3.3. Air Transmission Behaviour. Air permeability of the fabric samples was measured using TEXTEST FX 3300 air permeability tester. The testing was carried out using test area $5.08 \mathrm{~cm}^{2}$ and test pressure $100 \mathrm{~Pa}$ as per ASTM D737. An average of 30 observations is reported here.

3.4. Relative Water Vapour Permeability. The Permetest instrument was used to evaluate the relative moisture vapour transmission as per ISO 11092 as used by Nida and Arzu [4]. The instrument consists of a heated porous membrane to imitate sweating skin. An air current was used to remove the microclimate that is generated on top of membrane. The required heat for water evaporation from membrane with and without specimen was measured to calculate the relative water vapour permeability. In presence of fabric samples less heat is required because fabric creates a resistance to evaporation.

Relative water vapour permeability $\left(P_{\mathrm{wv}}\right)$

$$
\begin{aligned}
& =\frac{\text { heat loss with fabric on measuring head }}{\text { heat loss from bare measuring head }} \\
& =\frac{q_{1}}{q_{0}}=\frac{u_{1}}{u_{0}}
\end{aligned}
$$

where $u_{1}$ is the output voltage when fabric is placed on the measuring head and $u_{0}$ is the output voltage from bare measuring head.

3.5. Capillary Rise Measurement. The lab made wicking tester was used for capillary rise evaluation similar to YG (B) 871 capillometer as per BS3424 method 21(1973) [5,6]. The 20 $\times 2.5 \mathrm{~cm}$ samples were hanged vertically with lower $3 \mathrm{~cm}$ length dipped in $2 \%$ cold reactive dye solution [7]. Height was recorded after 10 minutes of water rise, and average height of 10 values is presented here.

\section{Result and Discussion}

\subsection{Thermal Behaviour}

4.1.1. Peak Heat Flow Density $\left(q_{\max }\right)$ of Fabric Samples. $q_{\max }$ is referred to as the measurement of warm cool feeling of a fabric when it comes in contact of human skin. According to Hes, $q_{\max }$ is the peak heat flow density between human skin and fabric. It feels at the time of instant contact of fabric with human skin [8-11]. The results for peak heat flow density are compiled in Table 2.

The value of $q_{\max }$ is the highest among the understudied fabric samples in case of highly dense compact spun filling yarn containing fabric sample $40 \mathrm{CtF} 108$ (i.e., $0.491 \mathrm{~K} \mathrm{~W} \mathrm{~m}^{2}$ ) which may be attributed to the fabric sample $40 \mathrm{CtF} 108$ having compact spun filling yarn with higher surface area and smoothness. The $q_{\max }$ value of sample $30 \mathrm{CoF} 108$ is higher than $30 \mathrm{CoF} 88$ which indicates that fabric cool touch effect can be improved by increasing the fabric compactness. This hypothesis is again strengthened by comparing the $q_{\max }$ value of $40 \mathrm{CtF} 88$ and $40 \mathrm{CtF} 108$ where the value of $40 \mathrm{CtF} 108$ exhibited higher $q_{\max }$ than 40CtF88. Similar trend is found in case of carded filling yarn by comparing the $q_{\max }$ value 
TABLE 2: Thermal properties of samples.

\begin{tabular}{|c|c|c|c|c|c|c|}
\hline S. no. & Sample code & $\begin{array}{c}\text { Thermal conductivity } \\
\text { coefficient, } \lambda \\
\mathrm{W} \mathrm{m}{ }^{-1} \mathrm{~K}^{-1}\end{array}$ & $\begin{array}{c}\text { Thermal diffusability, } \\
a \\
\mathrm{~m}^{-2} \mathrm{~s}^{-1}\end{array}$ & $\begin{array}{c}\text { Thermal absorbability, } \\
b \\
\mathrm{~W} \mathrm{~s}^{1 / 2} \mathrm{~m}^{-2} \mathrm{~K}^{-1}\end{array}$ & $\begin{array}{c}\text { Thermal resistance } \\
r \\
\mathrm{~m}^{2} \mathrm{KW}^{-1}\end{array}$ & $\begin{array}{l}\text { Peak heat flow density, } \\
\qquad q_{\max }\end{array}$ \\
\hline 1 & 30CaF88 & 19.81 & 0.051 & 63.82 & 26.75 & 0.368 \\
\hline 2 & 30CaF108 & 19.55 & 0.053 & 62.45 & 25.25 & 0.302 \\
\hline 3 & $40 \mathrm{CaF} 88$ & 19.62 & 0.046 & 62.14 & 26.21 & 0.387 \\
\hline 4 & 40CaF108 & 20.11 & 0.051 & 62.05 & & 0.356 \\
\hline 5 & $30 \mathrm{CoF} 88$ & 20.62 & 0.058 & 65.31 & 26.21 & 0.389 \\
\hline 6 & $30 \mathrm{CoF} 108$ & 23.88 & 0.062 & 64.99 & 26.36 & 0.399 \\
\hline 7 & $40 \mathrm{CoF} 88$ & 21.96 & 0.053 & 64.53 & 28.12 & 0.402 \\
\hline 8 & $40 \mathrm{CoF} 108$ & 24.18 & 0.055 & 63.83 & 29.33 & 0.423 \\
\hline 9 & $30 \mathrm{CtF} 88$ & 21.56 & 0.060 & 66.19 & 38.76 & 0.404 \\
\hline 10 & $30 \mathrm{CtF} 108$ & 23.12 & 0.066 & 65.91 & 30.24 & 0.426 \\
\hline 11 & $40 \mathrm{CtF} 88$ & 25.28 & 0.057 & 64.95 & 30.87 & 0.476 \\
\hline 12 & $40 \mathrm{CtF} 108$ & 22.92 & 0.059 & 64.13 & 33.25 & 0.491 \\
\hline
\end{tabular}

of $30 \mathrm{CaF} 88$ with $30 \mathrm{CaF} 108$ and $40 \mathrm{CaF} 88$ with $40 \mathrm{CaF}$ 108. Understanding the effect of fibre configuration in yarn structure on warm/cool touch of fabric comparison was made among the $30 \mathrm{CoF} 88,30 \mathrm{CtF} 88,30 \mathrm{CaF} 88$ and $40 \mathrm{CoF} 108$, and 40CtF108, 40CaF108. It is reflected from Figure 1 that the fibre configuration inside the yarn body is able to change the value of $q_{\max }$. More compact yarn will be cooler in touch. Finally yarn can be graded in increasing order of cool feel, carded, combed, and compact. The above discussion gives ample support to say safely that the yarn structure, yarn linear density, and yarn surface profile are effective tools to manipulate the warm cool feel of a fabric.

4.1.2. Thermal Conductivity of Fabric Samples. The thermal conductivity measurement by Alambeta is based on following principle [12]:

$$
\lambda=\frac{Q}{F \tau(\Delta T / \sigma)}\left(\mathrm{W} \mathrm{m}^{-1} \mathrm{~K}^{-1}\right),
$$

where $Q$ is the amount of conducted heat, $F$ is the area through which heat is conducted, $\tau$ is the time of heat conducting, $\Delta T$ drop of temperature, and $\sigma$ is the fabric thickness. Thermal conductivity data are given in Table 2 , and its dependence on weft yarn configurations is pictorially illustrated in Figure 2. It is reflected from the data that the yarn configuration which is influenced by the yarn manufacturing technique influence the $\lambda$. It is clear from Figure 2 that the thermal conductivity increases from carded to compact as yarn compactness increases by collapsing of free space among the constituent fibres. Similar types of results are verified in case of $40 \mathrm{~s} \mathrm{Ne}$ warps with different weft densities of carded, combed, and compact filling yarns.

4.1.3. Thermal Diffusion of Fabric Samples. The thermal diffusion measurement by Alambeta is based on the following equation:

$$
a=\frac{\lambda}{\rho c}\left(\mathrm{~m}^{-2} \mathrm{~s}^{-1}\right)
$$

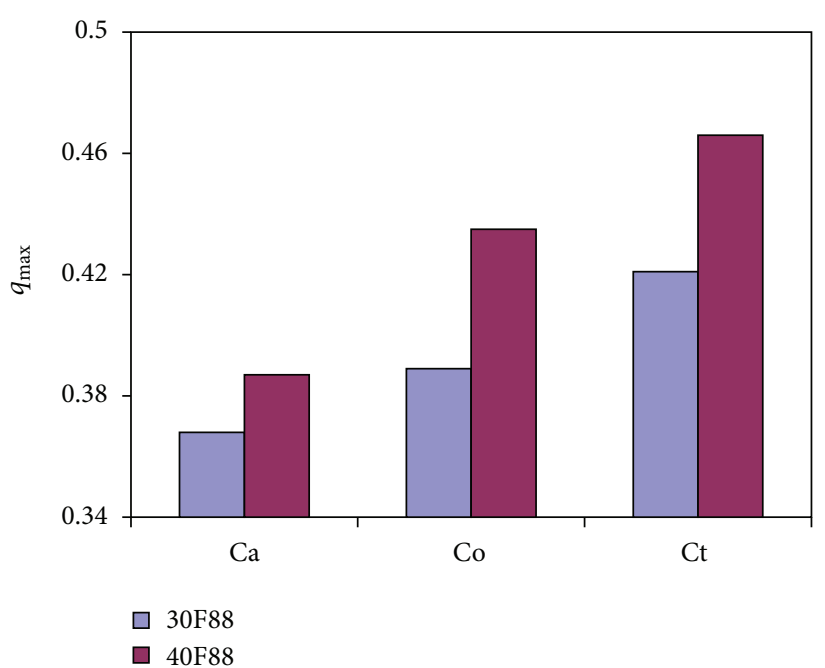

FIGURE 1: Effect of yarn count and fibre configuration on $q_{\max }$.

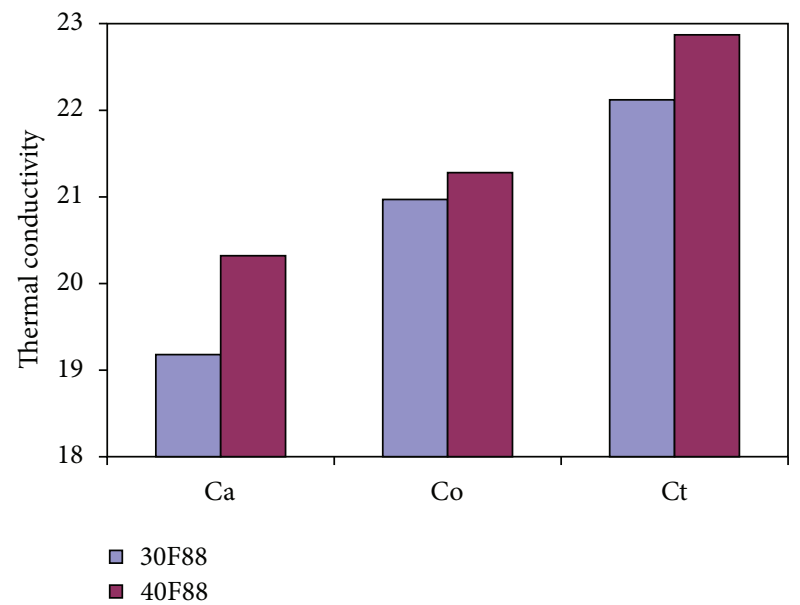

FIgURE 2: Effect of yarn count and fibre configuration on thermal conductivity. 
where $\rho$ is the fabric density, $c$ is the specific heat of fabric and $\lambda$ is the heat conductivity. The heat flow through textile structure is a function of fabric structure, fibre characteristics, and yarn configuration. Table 2 shows that the change in thermal diffusibility and other thermal aspects is dependent on yarn configuration which is different in carded, combed, and compact ring spun yarns as shown in Figure 3. Thermal diffusability increases from carded to compact. The free space increases from compact to combed to carded fabric sample as shown in Figure 8 by means of some representative photographs. This trend is repeated in both $30 \mathrm{~s} \mathrm{Ne}$ and $40 \mathrm{~s} \mathrm{Ne}$ warps when weft densities were changed from 88 to 108 for all three carded, combed, and compact yarns.

4.1.4. Thermal Resistance of Fabric Samples. The thermal resistance is the consequence of fabric thickness and thermal conductivity of fibrous material [12]. The following concept is opted in Alambeta:

$$
R=\frac{\sigma}{\lambda}\left(\mathrm{m}^{2} \mathrm{KW}^{-1}\right),
$$

where: $\sigma$ is the fabric thickness, $\lambda$ is the thermal conductivity.

The thermal resistance is a key parameter to establish thermal insulation behaviour of fabrics.

When fabric set is kept identical, fabric thickness is largely governed by fibre packing arrangement in yarn body. The fibre migration inside the yarn body will depend on the type of yarn like carded, combed, and compact as ideally shown in Figure 4 and practically shown in Figure 7. As the number of filling yarns increases in the fabric samples, the fabric thickness increases which is exhibited by the thermal resistance values of 30CaF88 and 30CoF108. Fabric samples which are manufactured with carded weft yarns, registered higher thermal resistance than fabric made of combed and compact ring spun yarns. This can be explained on the basis of fact that thermal resistance of yarns is primly influenced by the amount of air trapped in yarn body. The compact yarn configuration has the least trapped air as ideally shown in Figure 5 and actually in Figure 7.

4.1.5. Thermal Absorption of Fabric Samples. Thermal absorption measurement is based on the expression $[13,14]$

$$
b=\sqrt{\lambda \cdot \rho \times c}\left(\mathrm{~W} \mathrm{~s}^{1 / 2} \mathrm{~m}^{-2} \mathrm{~K}^{-1}\right),
$$

where $\lambda$ is the thermal conductivity, $\rho$ is the fabric density and $c$ is the specific heat of the fabric. The low value of thermal absorption gives a warm feeling [13, 14]. Thermal absorption values for different fabric samples are given in Table 2. Thermal absorption trend for various fabric samples is shown in Figures 5 and 6 . The fabric samples consist carded weft yarn shown lowest thermal absorbability than combed and compact weft yarn based fabric samples. It indicates that carded weft yarn based fabrics have stronger warm feeling than combed and compact weft yarn based fabrics in both $30 \mathrm{~s} \mathrm{Ne}$ and 40s Ne warp yarn samples as graphically shown in Figure 7. This may be based on the fact that carded yarn has the highest free space for an insulating material, that is, air, and lowest in compact yarn.

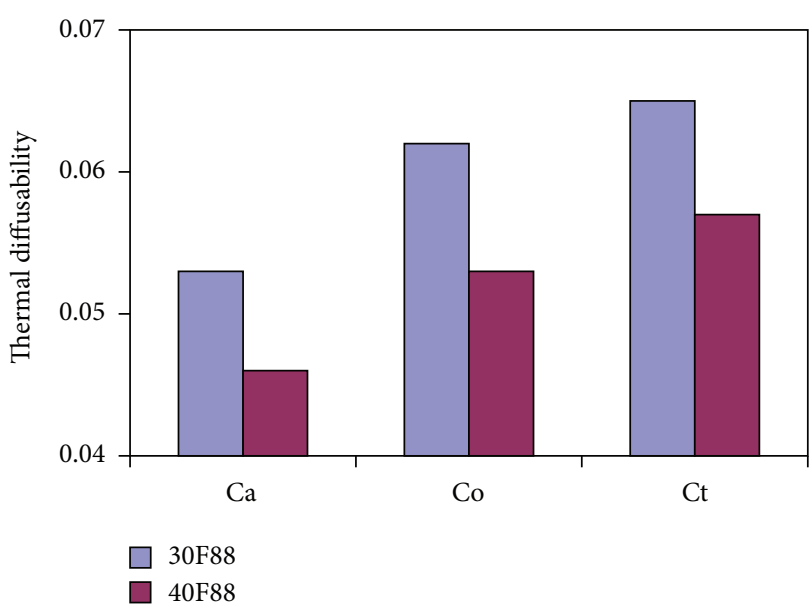

FIGURE 3: Effect of yarn type on thermal diffusion.

4.2. Air Permeability of Fabric Samples. The air permeability is expressed by the following relationship:

$$
A=\frac{V}{F \cdot \tau(\Delta p)}, \mathrm{dm}^{3} \mathrm{~m}^{-2} \mathrm{~s}^{-1},
$$

where: $V$ is the capacity of the flowing medium, $F$ is the area through which the medium is flowing, $\tau$ is the time flow and $\Delta p$ is the drop in pressure of the medium. The flow of omitted gases from human body to the environment and inward flow of fresh air towards human body make the air permeability a hygienic character of textiles. The air permeability of fabric depends on its porosity. The air permeability data are given in Table 3. Data indicates that by changing the amount and length of hairs on yarn surfaces, the air permeability of fabric samples changes [15]. In consequence of alteration in fibre configuration in yarn body (carded, combed, and compact yarns), air permeability of fabric samples changes [16].

The carded yarn offers higher drag resistance against the flow of air stream than combed and compact yarns. The fabric samples consisting of compact yarn has the highest air permeability due to less number of hairs on surface of most compact yarn body than carded and combed filling yarns. It is evident from air permeability data of Table 3 that, as the weft yarn density increases in all three types of yarns, the air permeability decreases. This may be attributed to the fact that increased density of filling yarns increases the total drag against air flow by increasing the available specific surface area. In consequence of higher specific surface area of each filament available specific surface in given fabric volume will increase and offer higher drag resistance to air.

4.3. Wicking Behaviour. The wicking behaviour of various fabrics depends on the capillary action, primly on capillary rise in constituent yarns [17]. The wicking behaviour depends on number of capillaries, size of capillary, and size distribution [18]. All these factors are influenced by packing of fibres in the yarn body [19].

It can be seen that the warp way wicking height was always higher than weft way in all fabric samples consisting of carded, combed, and compact filling yarns. Table 3 shows 
TABLE 3: Transmission properties of samples.

\begin{tabular}{lcccc}
\hline S. no & Sample code & $\begin{array}{c}\text { Air Permeability } \\
\left(\mathrm{cm}^{3} / \mathrm{cm}^{2} / \mathrm{sec}\right)\end{array}$ & $\begin{array}{c}\text { Wicking height }(\mathrm{mm}) \\
\text { after } 10 \text { min }\end{array}$ & $\begin{array}{c}\text { Moisture vapour } \\
\text { transmission rate }(\%)\end{array}$ \\
\hline 1 & $30 \mathrm{CaF} 88$ & 11.58 & 388 & 52.68 \\
2 & $30 \mathrm{CaF108}$ & 10.34 & 380 & 50.13 \\
3 & $40 \mathrm{CaF} 88$ & 12.42 & 379 & 50.32 \\
4 & $40 \mathrm{CaF108}$ & 10.85 & 366 & 48.44 \\
5 & $30 \mathrm{CoF} 88$ & 12.53 & 489 & 54.34 \\
6 & $30 \mathrm{CoF} 108$ & 11.95 & 478 & 53.56 \\
7 & $40 \mathrm{CoF} 88$ & 13.13 & 439 & 53.12 \\
8 & $40 \mathrm{CoF} 108$ & 12.47 & 422 & 51.48 \\
9 & $30 \mathrm{CtF} 88$ & 13.26 & 408 & 57.04 \\
10 & $30 \mathrm{CtF} 108$ & 12.74 & 402 & 56.19 \\
11 & $40 \mathrm{CtF} 88$ & 13.84 & 393 & 54.34 \\
12 & $40 \mathrm{CtF} 108$ & 13.26 & 386 & 52.49 \\
\hline
\end{tabular}
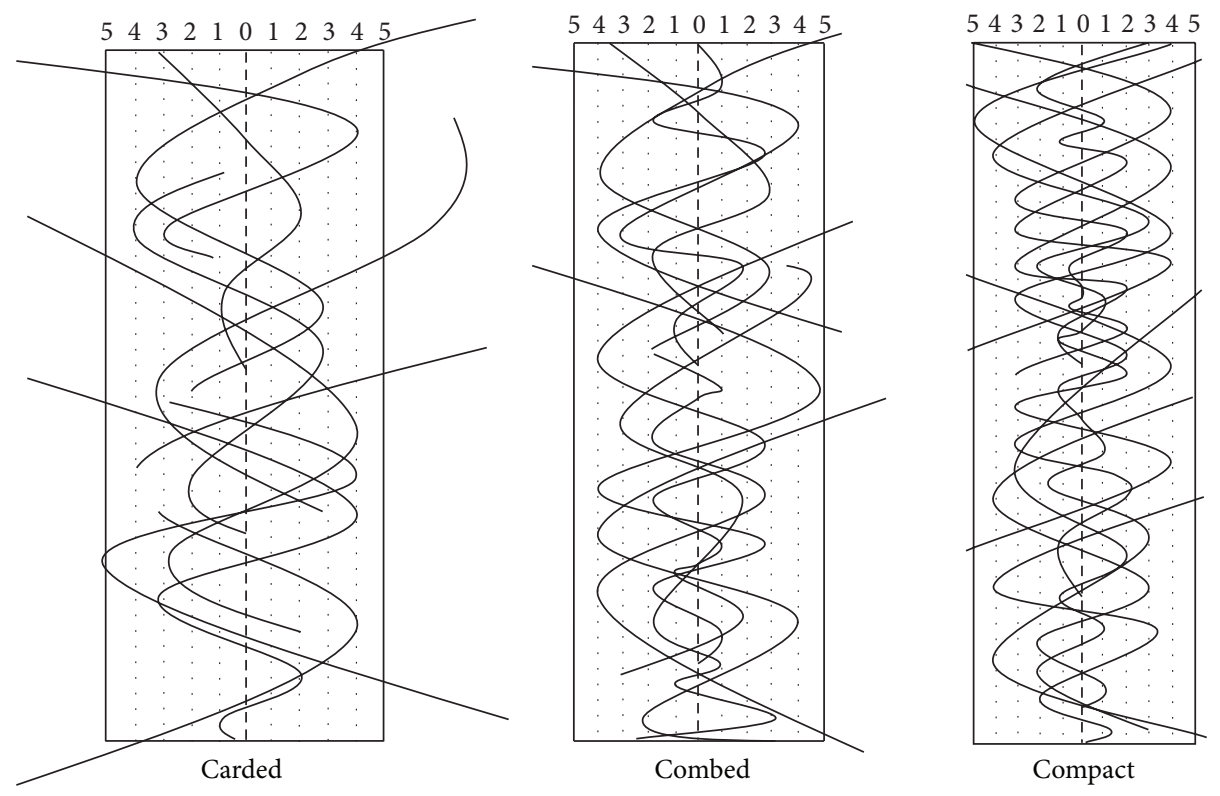

FIGURE 4: Ideal configuration of carded, combed and compact ring yarns.

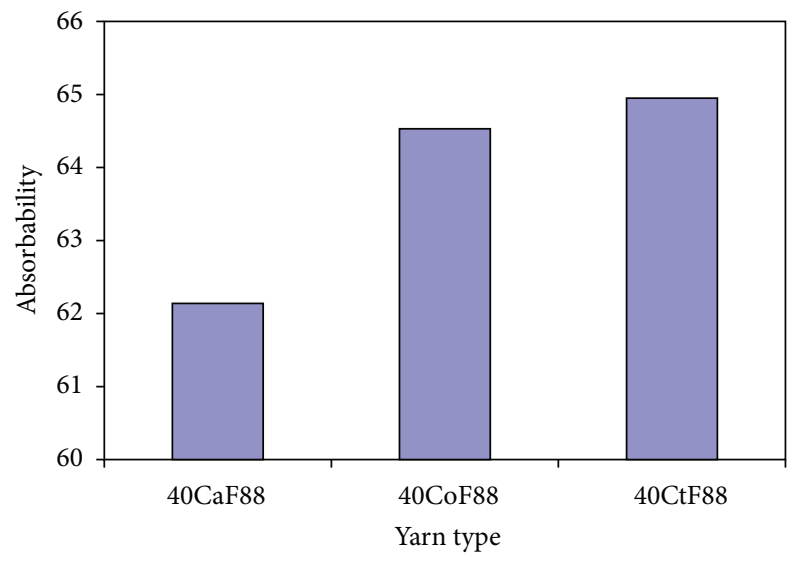

FIGURE 5: Effect of yarn type on absorbability.

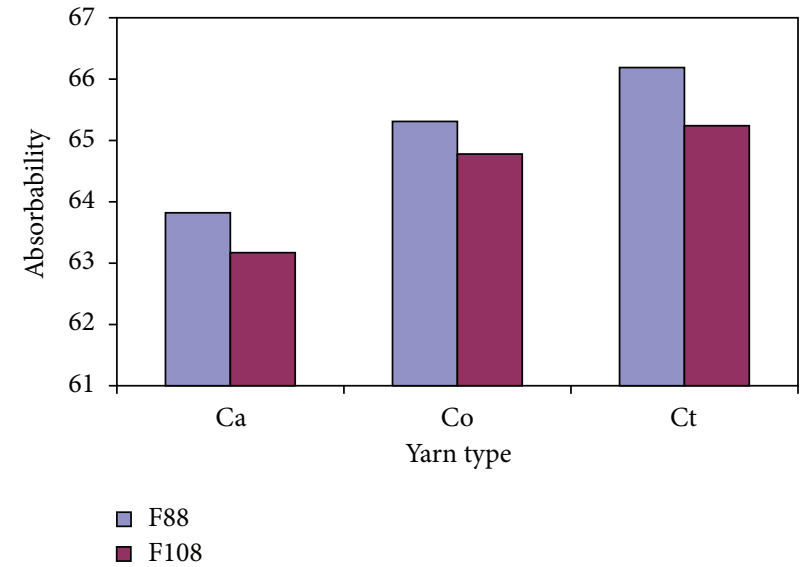

FIGURE 6: Effect of filling yarn density on absorbability. 


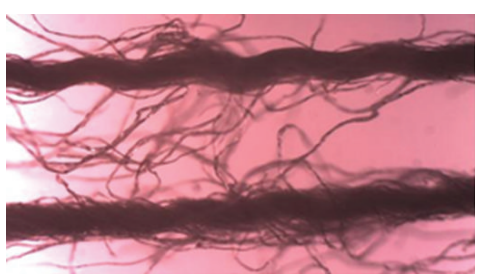

Carded yarn

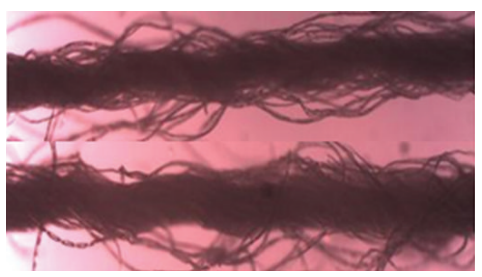

Combed yarn

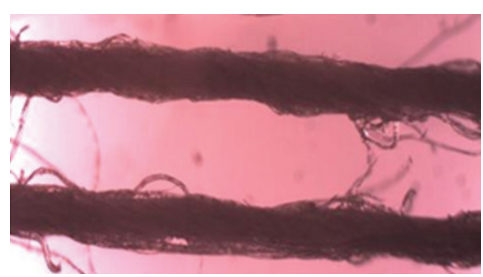

Compact yarn

FIGURE 7: Various types of ring yarns.

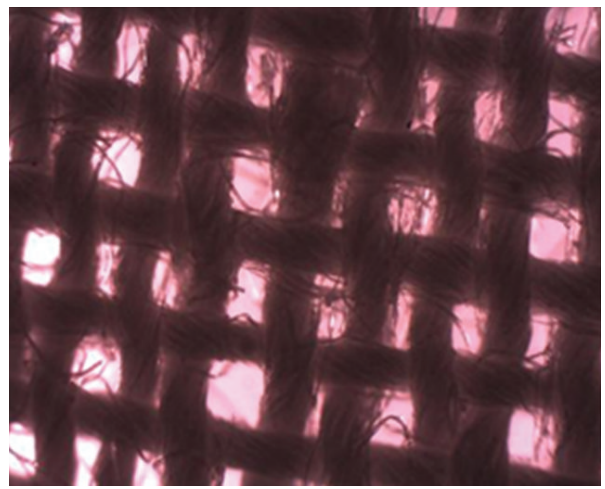

Fabric sample: $30 \mathrm{CaF} 88$

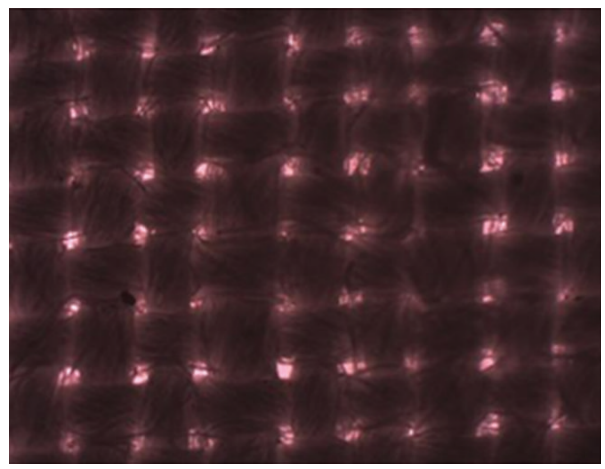

Fabric sample: $40 \mathrm{CtF} 108$

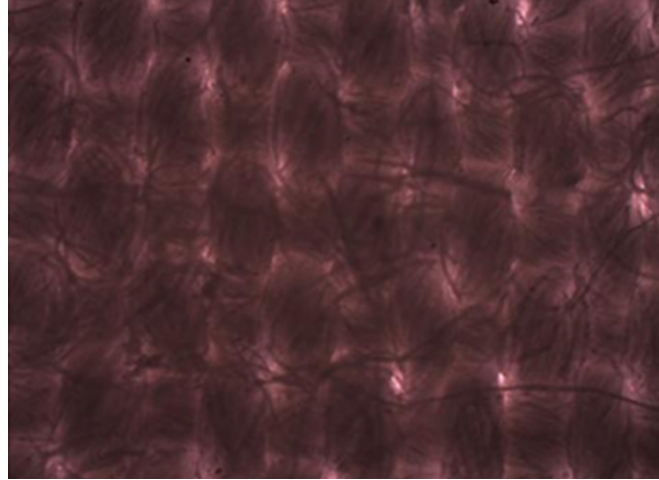

Fabric sample: 40CoF108

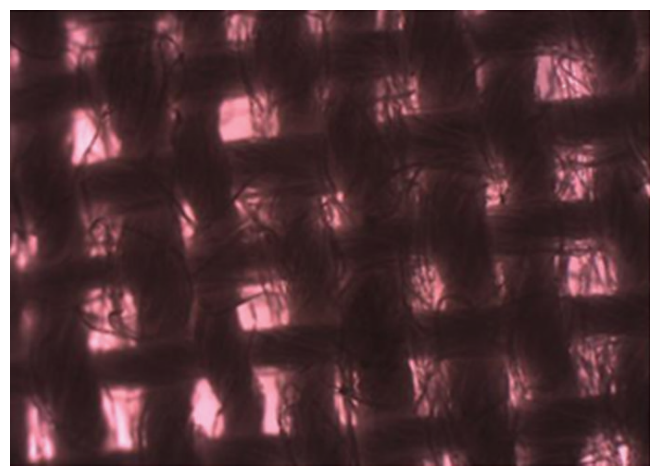

Fabric sample: $40 \mathrm{CaF} 88$

FIGURE 8: Some representative fabric samples.

that, as the number of filling yarns increases from 88 to 108 , wicking height decreases in all three cases of carded, combed, and compact yarns. The wicking height of combed filling yarns is higher than compact and carded yarn in both respective cases of 88 and 108 filling yarns per inch. This may be attributed to the fact that, as constituent fibres becomes straight in combed yarn from carded, wicking height increases because straight capillaries are available in case of combed yarn than carded yarn and consequently water rises a higher height in unit time. The wicking height decreases in case of compact filling yarn fabrics than combed. This is ascribed to the fact that as yarn compactness increases, some capillaries start to collapse, and this results in lower wicking height.

4.4. Water Vapour Permeability. Water vapour permeability (WVP) values of various specimens are given in Table 3. The results shows that three different configurations of ring yarn have different ranges of WVP values for the same set of fabrics. It is evident from Table 3 that WVP reduces as number of filling yarn increases from 88 to 108 in all three carded, combed, and compact configurations. This is ascribed to the fact that, as the number of filling yarns increases, fabric cover increases which suppresses the diffusibility of WVP fabric samples as similarly found by Kothari and Das Subhasis [20].

\section{Conclusions}

Various transmission behaviours of cotton shirting fabrics have been studied. It is inference from the study that compact spin yarns are able to manufacture a fabric of higher cool touch effect. Fabric samples made with compact weft yarns posses higher water vapour permeability than combed and carded weft yarn fabrics. Carded weft yarn based fabric samples shown higher resistance against air drag than combed and compact weft yarn filled fabric samples. Compact spun 
filling yarns are suitable to form shirting for summer wear for subcontinent. Carded yarns are able to manufacturer fabrics of higher thermal insulation suitable for winter wear.

\section{References}

[1] R. L. Barker, "From fabric hand to thermal comfort: the evolving role of objective measurements in explaining human comfort response to textiles," International Journal of Clothing Science and Technology, vol. 14, no. 3-4, pp. 181-200, 2002.

[2] C. V. Le, N. G. Ly, and R. Postle, "Heat and moisture transfer in textile assemblies. I. Steaming of wool, cotton, nylon and polyester fabric beds," Textile Research Journal, vol. 65, no. 4, pp. 203212, 1995.

[3] J. Fan and X. Cheng, "Heat and moisture transfer with sorption and phase change through clothing assemblies: part I: experimental investigation," Textile Research Journal, vol. 75, no. 2, pp. 99-105, 2005.

[4] O. Nida and M. Arzu, "Thermal comfort of some knitted structures," Fibres \& Textiles in Eastern Europe, vol. 15, no. 5, p. 94, 2007.

[5] P. R. Harnett and P. N. Mehta, "A survey and comparison of laboratory test methods for measuring wicking," Textile Research Journal, vol. 54, no. 7, pp. 471-478, 1984.

[6] Y.-L. Hsieh, A. Miller And, and J. Thompson, "Wetting, pore structure, and liquid retention of hydrolyzed polyester fabrics," Textile Research Journal, vol. 66, no. 1, pp. 1-10, 1996.

[7] T. Liu, K. Choi, and Y. Li, "Wicking in twisted yarns," Journal of Colloid and Interface Science, vol. 318, no. 1, pp. 134-139, 2008.

[8] I. Hes, Instruction Manual of the ALAMBETA Instrument, SENSORA, Liberec Registered Company, 1990.

[9] L. Hes, V. Hybil, and B. Bandyopadhyay, "Determination of warm/cool feeling of various fibrous polymers through thermal absorbtivity," Indian Journal of Fibre \& Textile Research, no. 16, p. 3, 1991.

[10] L. Hes, "Thermal properties of nonwovens," in Congress Index, vol. 87, Geneva, Switzerland, 1987.

[11] L. Hes, "New achievements in the area of the objective evaluation of thermal insulation and thermal-contact properties of textiles," in Proceedings of the 3rd Asian Textile Conference, vol. 2, pp. 1201-1203, 1995.

[12] I. Frydrych, G. Dziworska, and J. Bilska, "Comparative analysis of the thermal insulation properties of fabrics made of natural and man-made cellulose fibres," Fibres and Textiles in Eastern Europe, vol. 10, no. 4, pp. 40-44, 2002.

[13] N. Özdil, A. Marmarali, and S. D. Kretzschmar, "Effect of yarn properties on thermal comfort of knitted fabrics," International Journal of Thermal Sciences, vol. 46, no. 12, pp. 1318-1322, 2007.

[14] S. Kawabata, M. Niwa, and Y. Yamashita, "A guide line for manufacturing 'ideal fabrics"' International Journal of Clothing Science and Technology, vol. 11, no. 2-3, pp. 134-140, 1999.

[15] W. E. Morton and J. W. S. Hearle, "Forces in various directions," in Physical Properties of Textile Fibres, Chapter 17, pp. 399-402, The Textile Institute Publication, 3rd edition, 1993.

[16] B. Das, A. Das, V. K. Kothari, R. Fanguiero, and M. De Araújo, "Effect of fibre diameter and cross-sectional shape on moisture transmission through fabrics," Fibers and Polymers, vol. 9, no. 2, pp. 225-231, 2008.

[17] T. Ramchandran and N. Kesavaraja, "A Study on Influencing Factors for Wetting and Wicking Behaviour," Journal of The Institution of Engineers (India), vol. 84, no. 2, p. 39, 2004.
[18] J. Wiener and P. Dejlová, "Wicking and wetting in textiles," Autex Research Journal, vol. 3, no. 2, pp. 64-71, 2003.

[19] T. Liu, K. Choi, and Y. Li, "Wicking in twisted yarns," Journal of Colloid and Interface Science, vol. 318, no. 1, pp. 134-139, 2008.

[20] V. K. Kothari and Das Subhasis, "Moisture vapour transmission behaviour of cotton fabrics," Indian Journal of Fibre \& Textile Research, vol. 37, pp. 151-156, 2012. 

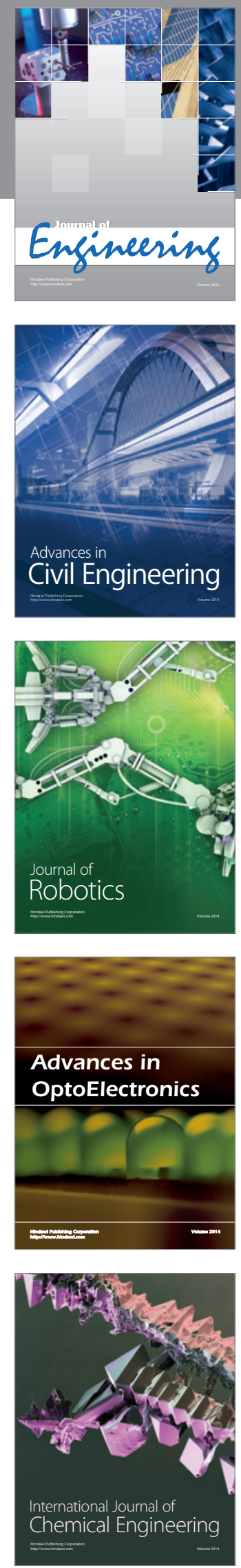

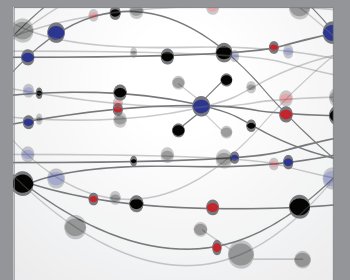

The Scientific World Journal
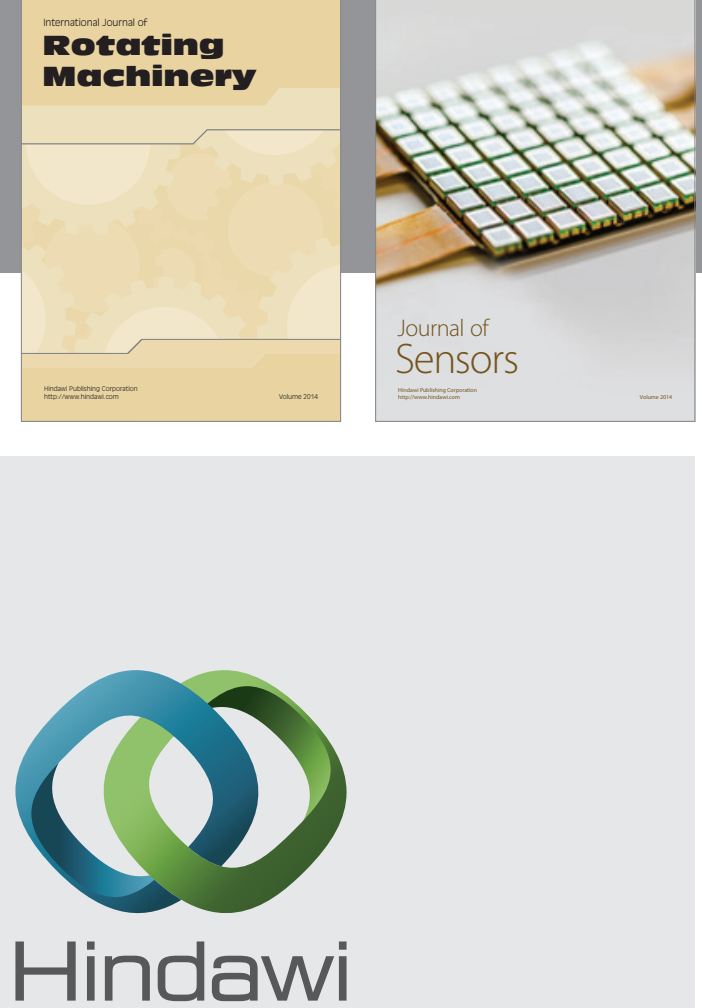

Submit your manuscripts at http://www.hindawi.com
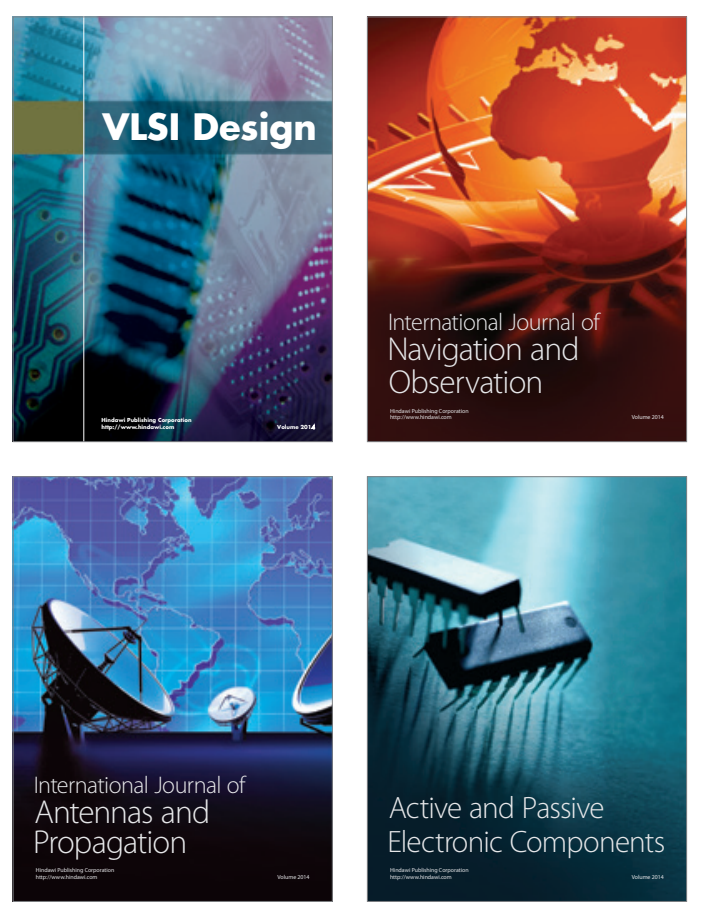
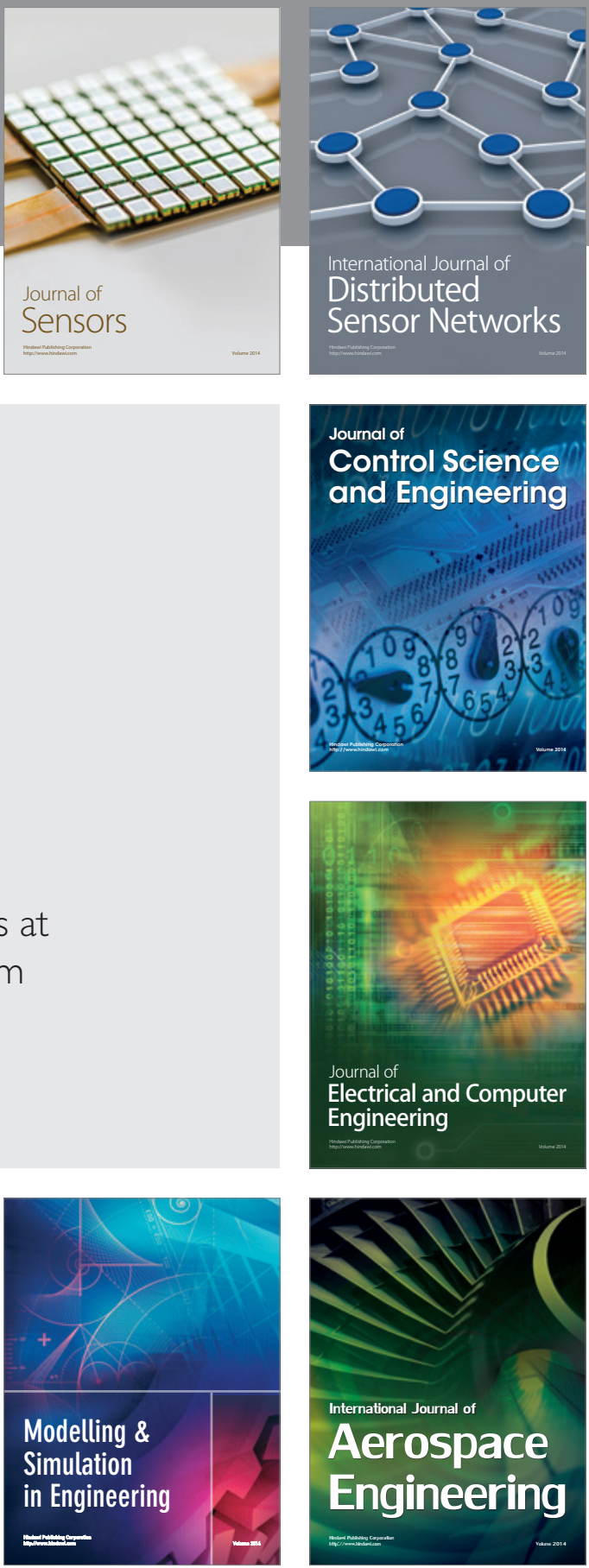

Journal of

Control Science

and Engineering
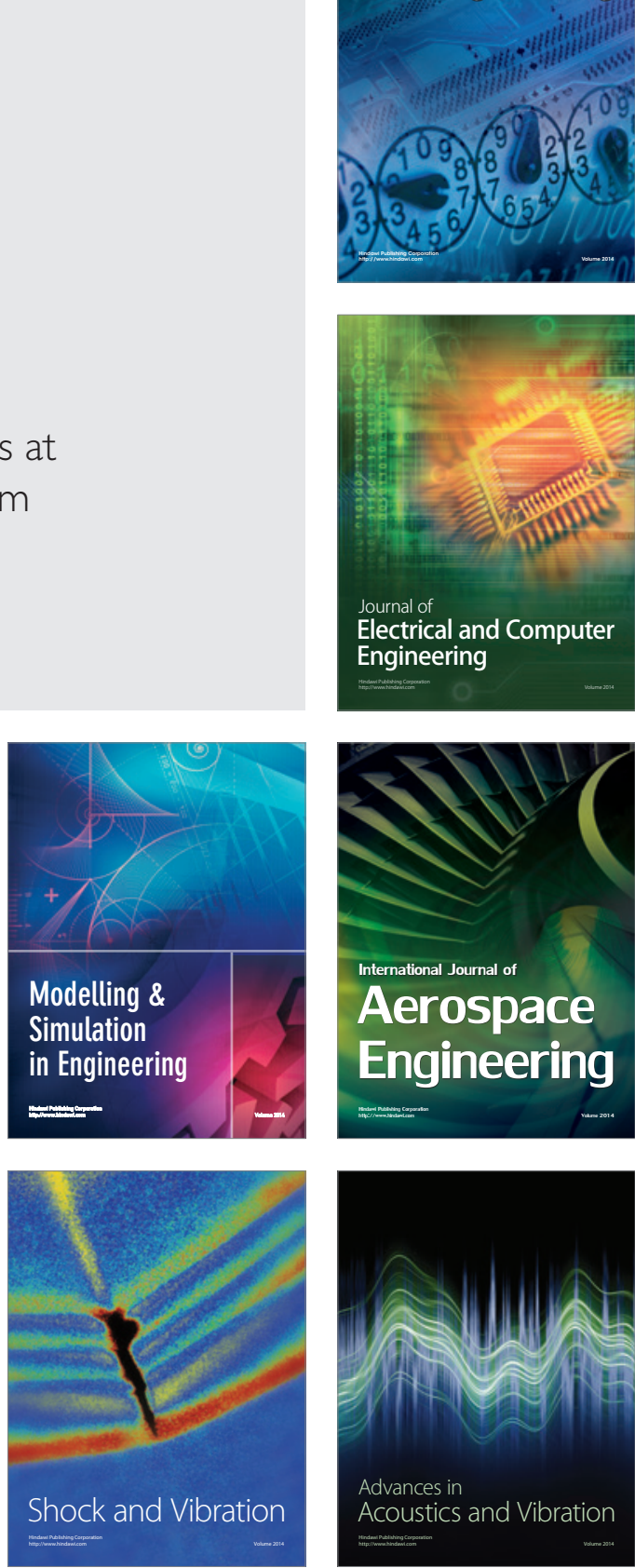